\title{
Cognition and fatigue in multiple sclerosis: Potential effects of medications with central nervous system activity
}

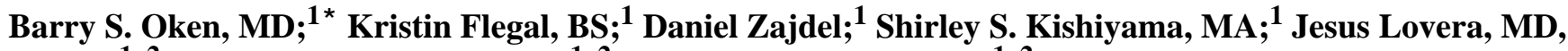 \\ MSPH; ${ }^{1-2}$ Bridget Bagert, MD, MPH; ${ }^{1-2}$ Dennis N. Bourdette, MD $^{1-2}$ \\ ${ }^{1}$ Department of Neurology, Oregon Health \& Science University, Portland, OR; ${ }^{2}$ Department of Neurology, Portland \\ Department of Veterans Affairs Medical Center, Portland, OR
}

\begin{abstract}
To evaluate the potential effects of medications with central nervous system (CNS) activity on cognitive function and fatigue in multiple sclerosis (MS), we performed a retrospective analysis of medication use among 70 subjects with MS who were participating in a clinical trial for evaluation of the effects of yoga and exercise programs on cognition and fatigue. Among these MS subjects, $74 \%$ were taking at least one potentially CNS-active medication. These 70 subjects were divided into two groups: those taking at least one CNS-active medication $(n=52)$ and those not on any medications with potential CNS activity $(n=18)$. We compared assessments of cognitive function and fatigue using an analysis of covariance. MS subjects on CNS-active medication had greater impairment on measures of processing speed, sustained attention, and fatigue than those not on these medications. While these findings do not establish a causal relationship between medication use and cognitive impairment and fatigue, the data indicate that researchers need to control for use of CNS-active medications when conducting studies of cognitive impairment and fatigue in MS subjects.
\end{abstract}

Key words: alertness, attention, attentional shifting, central nervous system agents, cognition, divided attention, fatigue, multiple sclerosis, processing speed, reaction time, sustained attention.

\section{INTRODUCTION}

Many cognitive function changes occur with multiple sclerosis (MS) [1-2]. These cognitive changes may involve almost any area of cognition, although deficits in attention, including speed of processing, are particularly common [2]. People with attentional system deficits from MS, or other conditions such as frontal lobe lesions or aging, may have memory deficits secondary to the attentional problems [35]. These attentional systems, along with the interrelated alertness systems, are more affected by drugs than other aspects of cognitive function. This is especially true for drugs with direct effects on neurotransmitters that are involved in the relatively nonspecific cortical projection systems (acetylcholine, norepinephrine, dopamine, serotonin, and histamine) or widely distributed cortical neurotransmitters (gamma-aminobutyric acid). Thus, a confounding issue in the study of cognition in MS is the frequent presence of medications with central nervous system (CNS) activity.

Abbreviations: AED = antiepileptic drug, ANCOVA = analysis of covariance, CESD-10 = Center for Epidemiologic Studies Depression Scale 10-item short form, CNS = central nervous system, EDSS = Expanded Disability Status Scale, MANCOVA = multivariate analysis of covariance, $\mathrm{MS}=$ multiple sclerosis, OHSU $=$ Oregon Health \& Science University, PASAT $=$ Paced Auditory Serial Addition Test, POMS = Profile of Mood States, $\mathrm{RT}=$ reaction time, $\mathrm{SSRI}=$ selective serotonin reuptake inhibitor, $\mathrm{UFOV}=$ useful field of view, WAIS ${ }^{\circledR}-$ III $=$ Wechsler Adult Intelligence Scale ${ }^{\circledR}$ — Third Edition.

* Address all correspondence to Barry S. Oken MD; Department of Neurology, Oregon Health \& Science University, 3181 SW Sam Jackson Park Road, Portland, OR 97239; 503-494-8873; fax: 503-494-9520. Email: oken@ohsu.edu

DOI: 10.1682/JRRD.2004.11.0148 
Many CNS-active drugs are known to affect cognitive function [6-7]. People who may already experience some cognitive impairment such as MS patients, as well as elderly people and patients with traumatic brain injury, are at higher risk than most people for additional cognitive dysfunction from medications [6]. Among these drugs with negative effects on cognitive function, antiepileptic drugs (AEDs) may be the most clinically studied. All AEDs have some negative effects on cognitive function as assessed by blinded placebo-controlled studies in healthy subjects, although some relative differences exist among the AEDs in the magnitude of these effects [8]. One point of potential relevance to MS is that subjective complaints of cognitive dysfunction from AEDs often exceed deficits identified by more formal neuropsychological testing, although cognitive tests sensitive to the effects of AEDs may require greater effort [9-10]. Many other drugs used by people with MS may negatively affect cognitive function as assessed by controlled trials in healthy control subjects, patient groups, or animals. This list includes tricyclic antidepressants, anticholinergics, first generation antihistamines, baclofen, beta-blockers, amantadine, selective serotonin reuptake inhibitors (SSRIs), and benzodiazepines [6-7,11-21]. On the other hand, methylphenidate and related drugs (modafinil) improve some cognitive functions in healthy subjects [11,22-23].

In many studies of MS and cognition, including some clinical trials, relatively little attention has been paid to the issue of CNS-active drugs [24]. We performed this retrospective analysis of data from an MS clinical trial to ascertain the frequency of use of medications that may unfavorably alter cognition in MS patients and to begin to probe the degree to which these drugs may negatively affect cognitive function and fatigue.

\section{METHODS}

\section{Subjects}

Data collected at Oregon Health \& Science University (OHSU) from a recent MS clinical trial were analyzed. The $70 \mathrm{MS}$ subjects in this analysis were from a clinical trial that compared ambulatory MS subjects in a 6-month yoga and exercise program with healthy control subjects. The results of this clinical trial have been reported, and only data from the first evaluation prior to randomization to treatment arms were analyzed for this study [25].
The study had approval of the OHSU Institutional Review Board and all subjects provided written informed consent. For inclusion in the study, subjects were between 18 and 65 yr with a clinical diagnosis of MS [26]. For consistency in the exercise and yoga intervention, only subjects with an Expanded Disability Status Scale (EDSS) of 6.0 or less, i.e., able to walk $100 \mathrm{~m}$ with at most unilateral support [27], were enrolled. For this relatively shortterm, nonpharmaceutical trial, we included subjects with either relapsing-remitting or progressive MS subtypes. Subjects spoke English as their primary language. We screened prospective participants for other major medical problems with a medical history and physical examination to ensure the safety of the intervention and to exclude subjects with an underlying medical illness that may impair cognition. Subjects were excluded for any of the following reasons: insulin-dependent diabetes; uncontrolled hypertension; evidence of liver or kidney failure; significant lung disease; alcoholism or other drug abuse; symptoms or signs of congestive heart failure, ischemic heart disease, or significant valvular disease; or significant visual impairment, i.e., best-corrected visual acuity worse than 20/50 binocularly. Subjects did not have baseline testing within 30 days of an MS exacerbation.

\section{Medications}

For descriptive purposes, medications known to have potential CNS activity were grouped into classes, e.g., AEDs or SSRIs (Table 1). Subjects who were not taking CNS-active medications that might impair cognitive function were assigned to one group. Statins and diuretics, for example, were medications in this category. Recombinant interferon- $\beta$ was considered to not have CNS activity because cognitive impairments have been shown not to occur from the interferon- $\beta$ doses used to treat MS. Glatiramer acetate was also considered to not have CNS activity. Methylphenidate and modafinil were not considered to have CNS activity because they are more likely to improve measures of attention and fatigue in healthy subjects than to impair them. Second generation antihistamines that have very limited effects on cognitive function in healthy subjects were also considered to have no CNS activity. Subjects taking CNS-active medications with mild or infrequent side effects on cognition or fatigue (e.g., SSRIs [21], beta-blockers [20], anticholinergics for bladder dysfunction $[13,16]$ ) or drugs with more consistent or more marked negative effects on cognitive function or fatigue (e.g., benzodiazepines, tricyclic antidepressants) were assigned to a second group. 
Table 1.

Percentage of multiple sclerosis patients who were taking different drugs or classes of drugs $(N=70)$.

\begin{tabular}{lc}
\hline \multicolumn{1}{c}{ Drug/Class } & Percentage \\
\hline Selective Serotonin Reuptake Inhibitor & 29 \\
Antiepileptic & 17 \\
Baclofen & 16 \\
Other Psychoactive (bupropion, trazodone) & 17 \\
Amantidine & 17 \\
Anticholinergic & 13 \\
Modafinil, Methylphenidate & 8.6 \\
Tricyclic Antidepressant & 7.1 \\
Benzodiazepine & 8.6 \\
Other than Nonsteroidal Anti-Inflammatory & 5.7 \\
$\quad$ or Acetominophen & \\
Tizanidine & 5.7 \\
Ginkgo & 16 \\
Not Central Nervous System Active & 16 \\
\hline \hline
\end{tabular}

\section{Assessments}

\section{Cognitive Testing}

The cognitive assessments used in this study were chosen because of their sensitivity to the yoga or exercise intervention and were not specifically chosen for maximal detection of neurotoxicity from CNS-active medications or MS-related cognitive deficits. When choosing cognitive tests to evaluate attention and other cognitive functions, we know that no cognitive test can completely isolate a single aspect of cognitive function. For example, the Paced Auditory Serial Addition Test (PASAT) [28] is a reliable attention test that has been used extensively in MS research even though performance on the test correlates with tests of working memory, sustained attention, and arithmetic ability [29]. Additionally, we needed the testing duration to be less than $2 \mathrm{hr}$ to avoid excessive subject fatigue, and this limited our ability to use other reasonable cognitive tests.

\section{Alertness}

Alertness was measured with two self-reported subjective scales, the Stanford Sleepiness Scale [30] and the Profile of Mood States (POMS) [31], which have both been shown to be sensitive to drug effects $[11,32]$.

\section{Sustained and Focused Attention}

The PASAT was administered because MS alters performance on the PASAT, and it is frequently used in
MS studies [28,33]. Performance on the Stroop colorword test [34] was used because it is a measure of attention that is sensitive to frontal lobe function [35].

\section{Computerized Cognitive Assessments}

For the attentional shifting, divided attention, and reaction time (RT) tests, subjects were seated comfortably $70 \mathrm{~cm}$ in front of a $39 \mathrm{~cm}$-wide color television monitor in a sound-attenuated room. For some of the tasks, subjects were instructed to maintain eye fixation at the center of the screen. Eye position was monitored with online pointof-regard information from an infrared-corneal reflection system (Iscan, Cambridge, Massachusetts) and a closedcircuit television camera. If subjects moved their eyes from the central fixation point during testing, the examiner instructed them to refixate at the center of the screen.

Attentional Shifting. The covert orienting of spatial attention task compares RT when targets are validly cued, neutrally cued, invalidly cued, or not cued [32,36-37]. The paradigm consisted of a series of 180 images that each contained a single shape to the left or right of a central fixation point, which occurred equally on the left or right side usually after a centrally placed arrow pointing left or right. Median RTs were calculated for the four cue conditions, and the ability of the subject to shift attention, the invalid-valid RT difference, was calculated.

The other attentional shifting task was adapted from that used in the Cambridge Neuropsychological Test Automated Battery and is related to the Wisconsin Card Sorting Test. It allows attentional shifting to be broken down into three types: intradimensional, reversal, and extradimensional, with some anatomic localization for each demonstrated in human and nonhuman primates [38]. The outcome measure was the number of shifts performed correctly.

Divided Attention. A modified useful field of view (UFOV) [39] task was chosen as a divided attention test, since it has ecologic validity relative to driving ability and has been previously shown to be altered in people with MS [39-41]. Additionally, the test assesses processing speed relatively independently of the speed of motor response. We adapted the commercially available UFOV-Part 3, which requires discrimination between a central car or van and simultaneous detection of the location of a peripheral car placed within a field of inverted triangles. The adaptation allowed us to determine the precise temporal perceptual threshold; i.e., the minimum stimulus duration necessary for the subject to respond 
correctly 75 percent of the time. The test included an implementation of the best algorithm for parameter estimation by sequential testing for psychophysical threshold determination [42]. The decrease and increase of display time continued until the threshold (75\% accuracy) was crossed twice. In addition to the threshold determination, we calculated the number of errors made at durations above threshold and divided it by the number of trials above threshold to determine the percentage error rate at durations above threshold. This error rate reflects lapses in attention for stimulus processing, since the subject is known to be able to accurately process the stimuli at these durations most of the time.

Reaction Time. Simple and choice median RTs were measured. Stimuli appeared in the center of the screen separated by random intervals between 1 and $4 \mathrm{~s}$. For the simple RT we presented 30 circles and for the choice RT we presented 64 stimuli, equal numbers of numerals one and two. The subjects were instructed to push response button(s) as soon as the stimuli appeared.

\section{Other Neuropsychological Assessments}

Two other cognitive assessments possibly affected by drugs were also administered: a 10-word list learning task (delayed memory adjusted for immediate recall) [43] and the Wechsler Adult Intelligence Scale ${ }^{\circledR}$ — Third Edition (WAIS ${ }^{\circledR}$-III) letter-number sequencing for assessment of working memory [44]. The Nine-Hole Peg Test was also administered as part of the MS Functional Composite score.

\section{Fatigue}

Fatigue was assessed with the POMS fatigue and vigor subscales [31], the Multidimensional Fatigue Inventory [45], and the energy and fatigue (vitality) measures on the 36-item short-form (SF-36) health-related quality-of-life assessment [46]. Depression was assessed with the POMS and the Center for Epidemiologic Studies Depression Scale 10-item short form (CESD-10) [47].

\section{Analysis}

We performed analysis of covariance (ANCOVA) on the cognitive data and fatigue assessments and separated the subjects into those taking no CNS-active medications and those taking one or more CNS-active medications. EDSS and age were used as covariates if they were related to the outcome measures $(p<0.10)$. For the 10 cognitive and 9 fatigue measures, we performed a multi- variate analysis of covariance (MANCOVA) to establish the overall significance of the differences between the two drug-taking groups and to account for the use of multiple comparisons.

\section{RESULTS}

Drug use data were available for 70 subjects who had an EDSS score ranging from 1.0 to 6.0. Of the 70 subjects, 74 percent were taking at least one medication with CNS activity that may directly affect cognitive function (Table 1). These subjects took a mean of two CNS-active medications (range 1 to 5). The most common medication was an SSRI, but moderate numbers of subjects were taking AEDs (mostly gabapentin for pain), baclofen, amantadine, and anticholinergics (for bladder symptoms).

Demographic data are shown in Table 2. We performed an overall MANCOVA to compare the cognitive data and fatigue assessments between subjects taking at least one CNS-active medication and those not taking any CNS-active medications. EDSS score and age were considered possible covariates, but EDSS score was the only significant variable and the only one we used as a covariate. The cognitive and fatigue data from the individual measure comparisons are shown in Table 3, with one of the fatigue measures significant by conservative Bonferroni criteria. Covariance for EDSS score was especially relevant because a significant relationship existed between EDSS score and the drug categories, with subjects taking more medication having a higher mean EDSS than those taking less medication (Table 2).

The only cognitive tests that significantly differed $(p<$ $0.05)$ in the two groups were choice RT $(p=0.02)$ and the percent errors above threshold in the divided attention task

Table 2.

Characteristics of study population $(N=70)$. Mean \pm standard deviation unless otherwise noted.

\begin{tabular}{lccc}
\hline \multicolumn{1}{c}{ Variable } & $\begin{array}{c}\text { No CNS-Active } \\
\text { Drugs }(\boldsymbol{n}=\mathbf{1 8})\end{array}$ & $\begin{array}{c}\geq \mathbf{1} \text { CNS-Active } \\
\text { Drugs }(\boldsymbol{n}=\mathbf{5 2})\end{array}$ & p-Value* $^{*}$ \\
\hline Women (No.) & 17 & 49 & 0.97 \\
Age (yr) & $44.7 \pm 8.7$ & $50.3 \pm 8.2$ & 0.02 \\
EDSS & $2.4 \pm 1.1$ & $3.1 \pm 1.6$ & 0.04 \\
CESD-10 & $6.2 \pm 3.4$ & $10.3 \pm 5.2$ & 0.003 \\
\hline
\end{tabular}

${ }^{*} p$-value for sex is Pearson $\chi^{2}$; other $p$-values are $t$-test.

EDSS $=$ Expanded Disability Status Scale, CESD-10 $=$ Center for Epidemiologic Studies Depression Scale 10-item short form, CNS = central nervous system. 
Table 3.

Cognitive and fatigue measures in multiple sclerosis patients grouped by whether or not subjects were taking medications with central nervous system (CNS) side effects (mean \pm standard deviation and analysis of covariance results).

\begin{tabular}{|c|c|c|c|}
\hline Variable & No CNS-Active Drugs & $\geq 1$ CNS-Active Drugs & $p$-Value \\
\hline \multicolumn{4}{|l|}{ Cognitive Measures } \\
\hline Set Shifting (adapted CANTAB) & $9.6 \pm 1.7$ & $8.9 \pm 2.5$ & 0.27 \\
\hline Stroop Interference (s) & $8.6 \pm 3.8$ & $11.9 \pm 7.1$ & 0.07 \\
\hline PASAT & $44.0 \pm 10.1$ & $45.4 \pm 10.5$ & 0.65 \\
\hline Simple RT (ms) & $325 \pm 111$ & $378.9 \pm 139.1$ & 0.07 \\
\hline Choice RT (ms) & $485.3 \pm 68.5$ & $575.6 \pm 126.5$ & 0.02 \\
\hline Spatial Attention Shift (ms) & $48.2 \pm 65.3$ & $47.1 \pm 49.9$ & 0.94 \\
\hline Delayed Word Recall (\%) & $86.1 \pm 15.9$ & $86.8 \pm 26.6$ & 0.93 \\
\hline Letter-Number Sequencing & $11.0 \pm 2.4$ & $11.4 \pm 2.6$ & 0.08 \\
\hline \multicolumn{4}{|l|}{ Divided Attention (UFOV) } \\
\hline Threshold (ms) & $84.1 \pm 66.8$ & $106.4 \pm 127.8$ & 0.97 \\
\hline Errors Above Threshold (\%) & $3.7 \pm 3.1$ & $5.8 \pm 3.7$ & 0.03 \\
\hline Nine-Hole Peg (s) & $22.5 \pm 3.3$ & $24.5 \pm 4.6$ & 0.41 \\
\hline \multicolumn{4}{|l|}{ Fatigue Measures } \\
\hline \multicolumn{4}{|l|}{ Multidimensional Fatigue Inventory } \\
\hline General Fatigue & $12.7 \pm 3.8$ & $15.2 \pm 3.3$ & 0.003 \\
\hline Physical Fatigue & $12.2 \pm 3.8$ & $14.6 \pm 3.7$ & 0.07 \\
\hline Reduced Activity & $9.9 \pm 4.4$ & $12.6 \pm 4.1$ & 0.09 \\
\hline Reduced Motivation & $8.3 \pm 3.0$ & $10.4 \pm 3.2$ & 0.01 \\
\hline Mental Fatigue & $10.3 \pm 4.8$ & $11.6 \pm 4.6$ & 0.31 \\
\hline \multicolumn{4}{|l|}{ POMS } \\
\hline Vigor & $13.9 \pm 5.6$ & $12.9 \pm 5.3$ & 0.51 \\
\hline Fatigue & $8.9 \pm 7.0$ & $13.2 \pm 7.2$ & 0.03 \\
\hline SF-36 Vitality & $51.3 \pm 20.8$ & $38.1 \pm 18.5$ & 0.02 \\
\hline Stanford Sleepiness Scale & $2.0 \pm 0.8$ & $2.2 \pm 1.0$ & 0.53 \\
\hline
\end{tabular}

( $p=0.03$ ). The other cognitive tests (PASAT, simple RT, Nine-Hole Peg, word-list memory, covert orienting of spatial attention, and set shifting) were not significantly different in the two groups, although trends toward significance $(p<0.10)$ were noted for the Stroop color-word test, simple RT, and WAIS ${ }^{\circledR}$-III letter-number sequencing. The fatigue measures showed greater differences in the two groups than the cognitive measures, with most of the measures either statistically significant at $p<0.05$ or trending toward significance ( $p$-values $<0.10$ ). Exclusion of the 6 out of 70 subjects who were taking CNS stimulants did not significantly change the analysis. One major confounding factor with these drug analyses is the presence of depression. CESD-10 scores were significantly higher in the group taking CNS-active medications (mean 10.3 vs 6.2, $p=0.01$ ), and the CESD-10 scores had a significant correlation with the fatigue measures. While covariance for depression (POMS or CESD-10) did not alter the effect of the drug group on cognitive function, it did alter the effect on fatigue. Covariance for depression in the ANCOVA reduced the statistical significance of all the fatigue measure differences: the lowest $p$-value increased to 0.15 when covariance for depression was present.

\section{DISCUSSION}

The majority of MS patients in this study were taking medications known to have a negative effect on cognitive function, and they were taking an average of two of these medications. The most commonly used drugs were SSRIs, AEDs, and baclofen. A correlation existed between use of CNS-active medications and some measures of attention and fatigue. Because of the many factors interrelated with 
CNS-active drug use, including severity of disease, presence of depression, and presence of pain, which may all contribute to cognitive changes, we cannot determine cause and effect between use of CNS-active drugs and cognitive dysfunction in this retrospective study.

The cognitive tests most related to drug use were those dependent on sustained attention and processing speed. The slower choice RT in medicated subjects is probably not simply an effect on motor systems, since the divided attention task measure that also showed a difference was independent of a timed motor response and no difference was noted in the Nine-Hole Peg Test. The cognitive effects are not likely related to multiple comparisons since the overall MANCOVA was significant. Additionally, 2 of the 10 measures were significant at the 0.05 level and 3 more trended toward significance with $p$-values between 0.05 and 0.1 . For 5 of the 11 cognitive measures to have a $p$-value $<0.1$ on a random basis is very unlikely (binomial test, $p=0.003$ ). The specific cognitive functions that were related to drug usage require more effort and most have a time component. These types of tasks more than others are more likely to be affected by AEDs such as gabapentin, even though subjective complaints of cognitive dysfunction may exceed objective measures, even in healthy controls [10]. Given the number of different medications and the limited numbers of subjects, we did not have sufficient statistical power in the study to determine the complete extent of the drug effects and which drugs may have greater effects. More definitive results depend on larger, future studies.

Fatigue is a common and potentially disabling symptom in MS [48-50]. Some aspects of fatigue in MS relate to depression, but some are not clearly related to depression [25,48,51-52]. Fatigue in MS is relatively independent of disease severity as assessed by EDSS or magnetic resonance imaging [25,48,51,53]. Some CNS-active medications may produce complaints closely related to fatigue and, in this study, subjects taking CNS-active medications had greater complaints of fatigue than subjects taking none. This relationship could not be disentangled from the effect of depression because both depression and CNS-active medications, some of which treat depression, were related to fatigue. Whichever effect on fatigue is more significant, depression or medications, it remains important for the clinician and researcher evaluating fatigue to consider the effects of CNS-active medications.

\section{CONCLUSION}

The results of this study emphasize the importance of considering the effects of medications in clinical studies of cognitive function and fatigue in MS. Use of medications with CNS activity is typically controlled for in most clinical trials with Alzheimer's patients. Many, but not all, of these clinical trials allow stable doses of certain CNSactive agents such as SSRIs and beta-blockers. Subjects on other drugs with greater effects on cognitive function, such as tricyclic antidepressants and benzodiazepines, are more typically excluded. However, simple exclusion in MS clinical trials may not be practical because of the high frequency of use of CNS-active medications in people with MS. Some of the relatively stringent drug exclusion criteria that have been used for Alzheimer's disease trials may contribute to recruitment problems for clinical trials of cognitive dysfunction in MS [54]. Without these exclusions, though, we are unsure as to how much the change in cognitive function is related to the intervention and how much to a change in CNS-active medications. Use of CNS-active medications should be included as a covariate in data analysis of clinical trials that assess effects on cognition and fatigue. Cognitive and fatigue outcome data from future clinical trials in MS need to more clearly address the issue of concurrent CNS-active medication use.

\section{ACKNOWLEDGMENTS}

This material was based on work supported by the National Institutes of Health (grant P50AT00066 and M01RR000334), the Department of Veterans Affairs, the National Multiple Sclerosis Society, and the Nancy Davis Center Without Walls.

\section{REFERENCES}

1. Rao SM. Neurobehavioral aspects of multiple sclerosis. New York (NY): Oxford University Press; 1990. p. 1-288.

2. Bobholz JA, Rao SM. Cognitive dysfunction in multiple sclerosis: A review of recent developments. Curr Opin Neurol. 2003;16(3):283-88. [PMID: 12858063]

3. Alexander MP, Freedman M. Amnesia after anterior communicating aneurysm rupture. Neurology. 1984;34(6):752-57. [PMID: 6539437] 
4. Light LL. Memory and aging: Four hypotheses in search of data. Annu Rev Psychol. 1991;42:333-76. [PMID: 2018397]

5. Deffenbacher KA. Effects of arousal on everyday memory. Hum Perform. 1994;7(2):141-61.

6. Meador KJ. Cognitive side effects of medications. Neurol Clin. 1998;16(1):141-55. [PMID: 9421545]

7. Stein RA, Strickland TL. A review of the neuropsychological effects of commonly used prescription medications. Arch Clin Neuropsychol. 1998;13(3):259-84. [PMID: 14590642]

8. Ortinski P, Meador KJ. Cognitive side effects of antiepileptic drugs. Epilepsy Behav. 2004;5 Suppl 1:S60-65. [PMID: 14725848]

9. Meador KJ, Loring DW, Huh K, Gallagher BB, King DW. Comparative cognitive effects of anticonvulsants. Neurology. 1990;40(3 Pt 1):391-94. [PMID: 2314578]

10. Salinsky MC, Binder LM, Oken BS, Storzbach D, Aron CR, Dodrill CB. Effects of gabapentin and carbamazepine on the EEG and cognition in healthy volunteers. Epilepsia. 2002;43(5):482-90. [PMID: 12027908]

11. Oken BS, Kishiyama SS, Salinsky MC. Pharmacologically induced changes in arousal: Effects on behavioral and electrophysiologic measures of alertness and attention. Electroenchepalogr Clin Neurophysiol. 1995;95(5):359-71. [PMID: 7489665]

12. Swartzwelder HS, Tilson HA, McLamb RL, Wilson WA. Baclofen disrupts passive avoidance retention in rats. Psychopharmacology (Berl). 1987;92(3):398-401. [PMID: 2819919]

13. Donnellan CA, Fook L, McDonald P, Playfer JR. Oxybutynin and cognitive dysfunction. BMJ. 1997;315(7119):1363-64. [PMID: 9402781]

14. Ferrara P, D’Aleo CM, Tarquini E, Salvatore S, Salvaggio E. Side-effects of oral or intravesical oxybutynin chloride in children with spina bifida. BJU Int. 2001;87(7):674-78. [PMID: 11350411]

15. Minzenberg MJ, Poole JH, Benton C, Vinogradov S. Association of anticholinergic load with impairment of complex attention and memory in schizophrenia. Am J Psychiatry. 2004;161(1):116-24. [PMID: 14702259]

16. Mulsant BH, Pollock BG, Kirshner M, Shen C, Dodge H, Ganguli M. Serum anticholinergic activity in a communitybased sample of older adults: Relationship with cognitive performance. Arch Gen Psychiatry. 2003;60(2):198-203. [PMID: 12578438$]$

17. Hadj Tahar A, Blanchet PJ, Doyon J. Motor-learning impairment by amantadine in healthy volunteers. Neuropsychopharmacology. 2004;29(1):187-94. [PMID: 14532914]

18. Saber AJ, Cain DP. Combined beta-adrenergic and cholinergic antagonism produces behavioral and cognitive impairments in the water maze: Implications for Alzheimer disease and pharmacotherapy with beta-adrenergic antagonists. Neuropsychopharmacology. 2003;28(7):1247-56. [PMID: 12700678$]$
19. Cahill L, Prins B, Weber M, McGaugh JL. Beta-adrenergic activation and memory for emotional events. Nature. 1994; 371(6499):702-4. [PMID: 7935815]

20. Ko DT, Hebert PR, Coffey CS, Sedrakyan A, Curtis JP, Krumholz HM. Beta-blocker therapy and symptoms of depression, fatigue, and sexual dysfunction. JAMA. 2002; 288(3):351-57. [PMID: 12117400]

21. Furlan PM, Kallan MJ, Ten Have T, Pollock BG, Katz I, Lucki I. Cognitive and psychomotor effects of paroxetine and sertraline on healthy elderly volunteers. Am J Geriatr Psychiatry. 2001;9(4):429-38. [PMID: 11739070]

22. Turner DC, Robbins TW, Clark L, Aron AR, Dowson J, Sahakian BJ. Cognitive enhancing effects of modafinil in healthy volunteers. Psychopharmacology (Berl). 2003;165(3): 260-69. [PMID: 12417966]

23. Elliott R, Sahakian BJ, Matthews K, Bannerjea A, Rimmer J, Robbins TW. Effects of methylphenidate on spatial working memory and planning in healthy young adults. Psychopharmacology (Berl). 1997;131(2):196-206. [PMID: 9201809]

24. Fischer JS, Priore RL, Jacobs LD, Cookfair DL, Rudick RA, Herndon RM, Richert JR, Salazar AM, Goodkin DE, Granger CV, Simon JH, Grafman JH, Lezak MD, O’Reilly Hovey KM, Perkins KK, Barilla-Clark D, Shacter M, Shucard DW, Davidson AL, Wende KE, Bourdette DN, KooijmansCoutinho MF. Neuropsychological effects of interferon beta$1 \mathrm{a}$ in relapsing multiple sclerosis. Multiple Sclerosis Collaborative Research Group. Ann Neurol. 2000;48(6):885-92. [PMID: 11117545]

25. Oken BS, Kishiyama SS, Zajdel D, Bourdette DN, Carlsen J, Haas M, Hugos C, Kraemer DF, Lawrence J, Mass M. Randomized controlled trial of yoga and exercise in multiple sclerosis. Neurology. 2004;62(11):2058-64. [PMID: 15184614$]$

26. McDonald W, Compston A, Edan G, Goodkin D, Hartung HP, Lublin FD, McFarland HF, Paty DW, Polman CH, Reingold SC, Sandberg-Wolheim M, Sibley W, Thompson A, Van den Noort S, Weinshenker BY, Wolinsky JS. Recommended diagnostic criteria for multiple sclerosis: Guidelines from the International Panel on the diagnosis of multiple sclerosis. Ann Neurol. 2001;50(1):121-27. [PMID: 11456302]

27. Kurtzke JF. Rating neurological impairment in multiple sclerosis: An Expanded Disability Status Scale (EDSS). Neurology. 1983;33(11):1444-52. [PMID: 6685237]

28. Gronwall DM. Paced Auditory Serial-Addition Task: A measure of recovery from concussion. Percept Mot Skills. 1977;44(2):367-73. [PMID: 866038]

29. Sherman EMS, Strauss E, Spellacy F. Validity of the Paced Auditory Serial Addition Test (PASAT) in adults referred for neuropsychological assessment after head injury. Clin Neuropsychol. 1997;11:34-45.

30. Hoddes E, Zarcone V, Smythe H, Phillips R, Dement WC. Quantification of sleepiness: A new approach. Psychophysiology. 1973;10(4):431-36. [PMID: 4719486$]$ 
31. McNair DM. Manual for the Profile of Mood States. San Diego (CA): Educational and Industrial Testing Service; 1992. p. 1-40.

32. Griesar WS, Zajdel DP, Oken BS. Nicotine effects on alertness and spatial attention in non-smokers. Nicotine Tob Res. 2002;4(2):185-94. [PMID: 12028851]

33. Fischer JS, Rudick RA, Cutter GR, Reingold SC. The Multiple Sclerosis Functional Composite Measure (MSFC): an integrated approach to MS clinical outcome assessment. National MS Society Clinical Outcomes Assessment Task Force. Mult Scler. 1999;5(4):244-50. [PMID: 10467383]

34. Perret E. The left frontal lobe of man and the suppression of habitual responses in verbal categorical behavior. Neuropsychologia. 1974;12(3):323-30. [PMID: 4421777]

35. Pardo JV, Pardo PJ, Janer KW, Raichle ME. The anterior cingulate cortex mediates processing selection in the Stroop attentional conflict paradigm. Proc Natl Acad Sci USA. 1990;87(1):256-59. [PMID: 2296583]

36. Posner MI. Orienting of attention. Q J Exp Psychol. 1980; 32(1):3-25. [PMID: 7367577]

37. Oken BS, Kishiyama SS, Kaye JA, Howieson DB. Attention deficit in Alzheimer's disease is not simulated by an anticholinergic/anithistaminergic drug and is distinct from deficits in healthy aging. Neurology. 1994;44(4):657-62. [PMID: 8164820]

38. Dias R, Robbins TW, Roberts AC. Dissociation in prefrontal cortex of affective and attentional shifts. Nature. 1996; 380(6569):69-72. [PMID: 8598908]

39. Ball K, Roenker D. UFOV: Useful field of view. San Antonio (TX): The Psychological Corporation; 1999. p. 1-103.

40. Shawaryn MA, Schultheis MT, Garay E, Deluca J. Assessing functional status: Exploring the relationship between the multiple sclerosis functional composite and driving. Arch Phys Med Rehabil. 2002;83(8):1123-29. [PMID: 12161835]

41. Ball K, Owsley C, Sloane ME, Roenker DL, Bruni JR. Visual attention problems as a predictor of vehicle crashes in older drivers. Invest Ophthalmol Vis Sci. 1993;34(11): 3110-23. [PMID: 8407219]

42. Pentland A. Maximum likelihood estimation: The best PEST. Percept Psychophys. 1980;28(4):377-79. [PMID: 7465322]

43. Morris J, Heyman A, Mohs R, Hughes JP, Van Belle G, Fillenbaum G, Mellits ED, Clark C. The Consortium to Establish a Registry for Alzheimer's Disease (CERAD) Part 1. Clinical and neuropsychological assessment of Alzheimer's disease. Neurology. 1989;39(9):1159-65. [PMID: 2771064]
44. Wechsler D. Wechsler Adult Intelligence scale. 3rd ed. San Antonio (TX): The Psychological Corporation; 1997. p. 1-217.

45. Smets EM, Garssen B, Bonke B, De Haes JC. The Multidimensional Fatigue Inventory (MFI) psychometric qualities of an instrument to assess fatigue. J Psychosom Res. 1995;39(3):315-25. [PMID: 7636775]

46. Ware JF. SF-36 health survey: Manual and interpretation guide. Boston (MA): The Medical Outcomes Trust; 1993. p. 1-320.

47. Andresen EM, Malmgren JA, Carter WB, Patrick DL. Screening for depression in well older adults: Evaluation of a short form of the CES-D (Center for Epidemiologic Studies Depression Scale). Am J Prev Med. 1994;10(2):77-84. [PMID: 8037935]

48. Krupp LB, Alvarez LA, LaRocca NG, Scheinberg LC. Fatigue in multiple sclerosis. Arch Neurol. 1988;45(4): 435-37. [PMID: 3355400]

49. Freal JE, Kraft GH, Coryell JK. Symptomatic fatigue in multiple sclerosis. Arch Phys Med Rehabil. 1984;65(3): 135-38. [PMID: 6703889]

50. Branas P, Jordan R, Fry-Smith A, Burls A, Hyde C. Treatments for fatigue in multiple sclerosis: A rapid and systematic review. Health Technol Assess. 2000;4(27):1-61. [PMID: 11074395]

51. Bakshi R, Shaikh ZA, Miletich RS, Czernecki D, Dmochowski J, Henschel K, Janardhan V, Dubey N, Kinkel PR. Fatigue in multiple sclerosis and its relationship to depression and neurologic disability. Mult Scler. 2000;6(3):181-85. [PMID: 10871830]

52. Ford H, Trigwell P, Johnson M. The nature of fatigue in multiple sclerosis. J Psychosom Res. 1998;45(1 Spec No):33-38. [PMID: 9720853]

53. Van der Werf SP, Jongen PJ, Lycklama a Nijeholt GJ, Barkhof F, Hommes OR, Bleijenberg G. Fatigue in multiple sclerosis: Interrelations between fatigue complaints, cerebral MRI abnormalities and neurological disability. J Neurol Sci. 1998;160(2):164-70. [PMID: 9849800]

54. Krupp LB, Christodoulou C, Melville P, Elkins LE, Hussein M. Interventions to improve memory in MS: the Aricept in MS study (AIMS): 24 week data. Neurology. 2003; 60:A477.

Submitted for publication November 22, 2004. Accepted in revised form January 31, 2005. 